\title{
THE CHARACTERIZATIONS OF HARDY-SOBOLEV SPACES BY FRACTIONAL SQUARE FUNCTIONS RELATED TO SCHRÖDINGER OPERATORS
}

\author{
Jizheng Huang, Pengtao Li, Yu Liu and Jie Xin \\ Beijing University of Posts and Telecommunications, School of Science \\ Beijing 100876, P. R. China; hjzheng@163.com \\ Qingdao University, College of Mathematics \\ Qingdao, Shandong 266071, P. R. China; ptli@qdu.edu.cn \\ University of Science and Technology Beijing, School of Mathematics and Physics \\ Beijing 100083, P. R. China; liuyu75@pku.org.cn \\ Qufu Normal University, School of Mathematical Sciences \\ Qufu 273165, P. R. China; fdxinjie@sina.com
}

\begin{abstract}
Let $L=-\Delta+V$ be a Schrödinger operator, where the potential $V$ satisfies the reverse Hölder condition. In this paper, via the heat semigroup $e^{-t L}$ and the Poisson semigroup $e^{-t \sqrt{L}}$, we introduce several classes of fractional square functions associated with $L$ including the Litttlewood-Paley $g$-function, the area integral and the $g_{\lambda}^{*}$-function, respectively. By the regularities of semigroup, we establish several square function characterizations of the Hardy space and the Hardy-Sobolev space related to the Schrödinger operator.
\end{abstract}

\section{Introduction}

It is a well-known fact that, as a class of important Calderón-Zygmund singular integrals, the square functions are usually applied to characterize function spaces. In 1972, Fefferman and Stein [18] proved that the square functions can be used to characterize Hardy spaces $H^{p}\left(\mathbf{R}^{d}\right)$ for $0<p \leq 1$. From then on, such characterizations were extended to other settings. We refer the reader to $[13,11,21]$ and the references therein. In the last decades, many researchers pay their attentions to the equivalent characterizations of Hardy spaces associated with operators, such as $[16,13,21,6,26,25,29,38,7,39,40,41]$ and the references therein. In particular, Dziubański and Zienkiewicz [16] dealt with the Hardy space associated with the Schrödinger operator. In this paper, based on the fractional square functions, we will establish several equivalent characterizations of the Hardy space and the Hardy-Sobolev space associated with the Schrödinger operator.

In the following, we recall some backgrounds of the Hardy space and the HardySobolev space associated with the Schrödinger operator, respectively. Let $L=-\Delta+$ $V$ be a Schrödinger operator on $\mathbf{R}^{d}, d \geq 3$, where $V \not \equiv 0$ is a nonnegative potential. Assume that $V$ belongs to the reverse Hölder class $B_{q}, q \geq d / 2$, i.e.,

$$
\left(\frac{1}{|B|} \int_{B} V^{q}(x) d x\right)^{1 / q} \leq C\left(\frac{1}{|B|} \int_{B} V(x) d x\right) \text { for every ball } B .
$$

https://doi.org/10.5186/aasfm.2020.4530

2010 Mathematics Subject Classification: Primary 42B35, 47A60, 42B25.

Key words: Hardy space, Hardy-Sobolev spaces, Schrödinger operator, fractional square functions. 
Let $\left\{T_{t}^{L}\right\}_{t>0}$ be the semigroup of linear operators generated by $-L$ and let $K_{t}^{L}(\cdot, \cdot)$ be their kernels. Since $V$ is nonnegative, the Feynman-Kac formula implies that

$$
0 \leq K_{t}^{L}(x, y) \leq \widetilde{T}_{t}(x, y):=(4 \pi t)^{-d / 2} \exp \left(-|x-y|^{2} / 4 t\right) .
$$

Dziubański and Zienkiewicz [16] defined the Hardy space $H_{L}^{1}\left(\mathbf{R}^{d}\right)$ as

$$
H_{L}^{1}\left(\mathbf{R}^{d}\right)=\left\{f \in L^{1}\left(\mathbf{R}^{d}\right): M f \in L^{1}\left(\mathbf{R}^{d}\right)\right\},
$$

where

$$
M f(x)=\sup _{t>0}\left|T_{t}^{L} f(x)\right| .
$$

The Hardy space $H_{L}^{1}\left(\mathbf{R}^{d}\right)$ is a Banach space with the norm $\|f\|_{H_{L}^{1}}=\|M f\|_{L^{1}}$. In order to give the atomic decomposition of the Hardy spaces associated with $L$, we introduce the function

$$
\rho(x):=\sup \left\{r>0: \frac{1}{r^{d-2}} \int_{B(x, r)} V(y) d y \leq 1\right\} .
$$

Definition 1.1. For $1 \leq q \leq \infty$, a function $a$ is an $H_{L}^{q}$-atom associated with a ball $B\left(x_{0}, r\right)$ if

(i) $\operatorname{supp} a \subset B\left(x_{0}, r\right)$;

(ii) $\|a\|_{L^{q}} \leq\left|B\left(x_{0}, r\right)\right|^{1 / q-1}$;

(iii) if $r<\rho\left(x_{0}\right)$, then $\int a(x) d x=0$.

The atomic quasi-norm of $H_{L}^{1}\left(\mathbf{R}^{d}\right)$ is defined by $\|f\|_{L \text {-atom, } q}=\inf \left\{\sum\left|c_{j}\right|\right\}$, where the infimum is taken over all decompositions $f=\sum c_{j} a_{j}$ and $\left\{a_{j}\right\}$ are $H_{L}^{q}$-atoms.

We have the following $H_{L}^{q}$-atomic decomposition of $H_{L}^{1}\left(\mathbf{R}^{d}\right)$.

Proposition 1.2. Let $1 \leq q \leq \infty$. The norms $\|f\|_{H_{L}^{1}}$ and $\|f\|_{L \text {-atom,q }}$ are equivalent, i.e., there exists a constant $C>0$ such that

$$
C^{-1}\|f\|_{H_{L}^{1}} \leq\|f\|_{L \text {-atom }, q} \leq C\|f\|_{H_{L}^{1}} .
$$

Proof. The case $q=\infty$ is obtained by Dziubański and Zienkiewicz [16]. For $1 \leq q<\infty$, it is easy to see that any $H_{L}^{\infty}$-atom is also an $H_{L}^{q}$-atom. On the other hand, by Proposition 2.1, we can use a direct computation to deduce that there exists a constant $C$ such that for any $H_{L}^{q}$-atom $a,\|M(a)\|_{1} \leq C$. We omit the details.

Now, we can define the square function. For $k \in \mathbf{N}$, let

$$
Q_{t}^{k} f(x)=t^{2 k}\left(\left.\partial_{s}^{k} T_{s}^{L}\right|_{s=t^{2}} f\right)(x),
$$

then square function associated with $\left\{Q_{t}^{k}\right\}$ is defined as

$$
\left(S_{k}^{L}(f)\right)(x):=\left(\int_{0}^{+\infty} \int_{|x-y|<t}\left|Q_{t}^{k}(f)(y)\right|^{2} \frac{d y d t}{t^{d+1}}\right)^{1 / 2} .
$$

The following square function characterization was obtained by Hoffmann et al. (cf. [21]).

Proposition 1.3. Let $k \in \mathbf{N}$. A function $f \in H_{L}^{1}\left(\mathbf{R}^{d}\right)$ if and only if $f \in L^{1}\left(\mathbf{R}^{d}\right)$ and the square function $S_{L}^{k}(f) \in L^{1}\left(\mathbf{R}^{d}\right)$. Moreover,

$$
\|f\|_{H_{L}^{1}} \sim\left\|S_{L}^{k}(f)\right\|_{L^{1}}+\|f\|_{L^{1}}
$$


In order to define the fractional square function, we first introduce the Poisson semigroup. Let $\left\{P_{t}^{L}\right\}_{t>0}$ be the semigroup of linear operators generated by $-\sqrt{L}$. Denote by $P_{t}^{L}(\cdot, \cdot)$ the integral kernel of $P_{t}^{L}$. Since $V$ is nonnegative, it can be seen from the Feynman-Kac formula that $P_{t}^{L}(\cdot, \cdot)$ is dominated by the classical Poisson kernel $\widetilde{P}_{t}(\cdot, \cdot)$ :

$$
0 \leq P_{t}^{L}(x, y) \leq \widetilde{P}_{t}(x, y):=\frac{C_{d} t}{\left(t^{2}+|x-y|^{2}\right)^{(d+1) / 2}} .
$$

Segovia and Wheeden [34] studied several types of fractional square and Lusin functions associated with $e^{-t(-\Delta)^{1 / 2}}$. Betancor et al. in [5] introduced the fractional square functions associated with $P_{t}^{L}$. The fractional derivatives $\partial_{t}^{\alpha} P_{t}^{L}, \alpha>0$, are defined as follows (cf. [34]):

$$
\partial_{t}^{\alpha} P_{t}^{L}(x, y):=\frac{e^{-i \pi(m-\alpha)}}{\Gamma(m-\alpha)} \int_{0}^{\infty} \partial_{t}^{m} P_{t+s}^{L}(x, y) s^{m-\alpha} \frac{d s}{s}, \quad m=[\alpha]+1 .
$$

Using the Poisson semigroup $\left\{P_{t}^{L}\right\}_{t>0}$, we define the fractional square functions as follows:

$$
\left\{\begin{array}{l}
g_{\alpha}(f)(x):=\left(\int_{0}^{\infty}\left|t^{\alpha} \partial_{t}^{\alpha} P_{t}^{L} f(x)\right|^{2} \frac{d t}{t}\right)^{1 / 2}, \\
G_{\alpha}(f)(x):=\left(\int_{0}^{\infty} \int_{|x-y|<t}\left|t^{\alpha} \partial_{t}^{\alpha} P_{t}^{L} f(x)\right|^{2} \frac{d y d t}{t^{d+1}}\right)^{1 / 2}, \\
g_{\alpha ; \lambda}^{*}(f)(x):=\left(\int_{0}^{\infty} \int_{\mathbf{R}^{d}}\left(\frac{t}{|x-y|+t}\right)^{2 \lambda}\left|t^{\alpha} \partial_{t}^{\alpha} P_{t}^{L} f(x)\right|^{2} \frac{d y d t}{t^{d+1}}\right)^{1 / 2} .
\end{array}\right.
$$

The first main result of this paper is the following characterizations of the Hardy space $H_{L}^{1}\left(\mathbf{R}^{d}\right)$.

Theorem 1.4. Let $\alpha \geq \frac{1}{2}$ and $\lambda>d / 2$. The following assertions are equivalent:

(i) $f \in H_{L}^{1}\left(\mathbf{R}^{d}\right)$;

(ii) $f \in L^{1}\left(\mathbf{R}^{d}\right)$ and $g_{\alpha}(f) \in L^{1}\left(\mathbf{R}^{d}\right)$;

(iii) $f \in L^{1}\left(\mathbf{R}^{d}\right)$ and $G_{\alpha}(f) \in L^{1}\left(\mathbf{R}^{d}\right)$;

(iv) $f \in L^{1}\left(\mathbf{R}^{d}\right)$ and $g_{\alpha ; \lambda}^{*}(f) \in L^{1}\left(\mathbf{R}^{d}\right)$.

Moreover, for every $f \in H_{L}^{1}\left(\mathbf{R}^{d}\right)$,

$$
\|f\|_{H_{L}^{1}} \sim\|f\|_{1}+\left\|g_{\alpha}(f)\right\|_{1} \sim\|f\|_{1}+\left\|G_{\alpha}(f)\right\|_{1} \sim\|f\|_{1}+\left\|g_{\alpha ; \lambda}^{*}(f)\right\|_{1} .
$$

In what follows, we consider the Hardy-Sobolev space associated with the Schrödinger operator, which are also important in harmonic analysis due to the fact that they can be used to give the strong boundedness of some linear operators instead of the weak boundeness. Denote by $\mathcal{S}^{\prime}$ the space of tempered distributions and denote by $\mathcal{P}$ the space of polynomials, respectively. Let $I_{\alpha}: \mathcal{S}^{\prime} / \mathcal{P} \rightarrow \mathcal{S}^{\prime} / \mathcal{P}$ be the Bessel potential operator. The function space $I_{\alpha}\left(H^{p}\right)$ which is called Hardy-Sobolev spaces are natural generalizations of the homogeneous Sobolev space $I_{\alpha}\left(L^{p}\right)$ to the range $0<$ $p \leq 1$. Hardy-Sobolev spaces associated with the Laplacian were studied by many authors. In [35], Strichartz investigated the space $I_{\alpha}\left(H^{p}\right), 0<p \leq 1$, and proved that $I_{n / p}\left(H^{p}\right)$ was an algebra and found equivalent norms for the Hardy-Sobolev space or, more generally, for the corresponding space with fractional smoothness and Lebesgue exponents in the range $p>n /(n+1)$. The trace properties of the space $I_{\alpha}\left(H^{p}\right)$ were discussed by Torchinsky [36]. Miyachi [32] characterized the Hardy-Sobolev spaces in terms of maximal functions related to mean oscillation of the function in cubes. In particular, he obtained a counterpart of previous results of Calderón and of the general theory of De Vore and Sharpley [12]. 
Recently, many scholars pay their attentions to Hardy-Sobolev spaces and their variants on $\mathbf{R}^{d}$, or on subdomains. In [8], Chang, Dafni, and Stein studied HardySobolev spaces in connection with estimates for elliptic operators. Furthermore, Aucher, Emmanuel, and Tchamitchian [1] studied these spaces with applications to square roots of elliptic operators. A simple strictly pointwise characterization of the Hardy-Sobolev space in terms of first difference was obtained by Koskela and Saksman in [28]. Lou and Yang [30] gave the atomic decomposition of the HardySobolev space and proved the endpoint case of the div-curl theorem in [10]. More about Hardy-Sobolev spaces can be found in [20, 9, 24, 33]. In [3] and [4], the authors considered Hardy-Sobolev spaces on the manifold. In [22] and [23], Huang and his coauthors studied the Hardy-Sobolev spaces associated with Hermite and special Hermite operators.

Now, we give the definitions of Hardy-Sobolev spaces associated with the Schrödinger operator $L$.

Definition 1.5. For $\alpha>0$, the inhomogeneous Hardy-Sobolev space $H_{L}^{1, \alpha}\left(\mathbf{R}^{d}\right)$ is defined as the set of all functions $f \in H_{L}^{1}\left(\mathbf{R}^{d}\right)$ such that $(I+L)^{\alpha / 2} f \in H_{L}^{1}\left(\mathbf{R}^{d}\right)$ with the norm

$$
\|f\|_{H_{L}^{1, \alpha}}:=\left\|(I+L)^{\alpha / 2} f\right\|_{H_{L}^{1}}+\|f\|_{H_{L}^{1}} .
$$

In 1969, Segovia and Wheeden [34] obtained the characterization of the Sobolev space $L^{p, \alpha}$ via the fractional square function defined as

$$
g_{k, \alpha}(f)=\left(\int_{0}^{\infty}\left|t^{k-\alpha} \frac{\partial^{k} \widetilde{P}_{t} f}{\partial t^{k}}\right|^{2} \frac{d t}{t}\right)^{1 / 2}, \quad k \geq \alpha>0 .
$$

In [5], the authors introduced the fractional square functions in the case of the Schrödinger operator $L$ and characterized the potential spaces associated with $L$. The fractional Littlewood-Paley $g$-function associated with $\left\{P_{t}^{L}\right\}_{t>0}$ is defined as

$$
g_{k, \alpha}(f)=\left(\int_{0}^{\infty}\left|t^{k-\alpha} \frac{\partial^{k} P_{t}^{L} f}{\partial t^{k}}\right|^{2} \frac{d t}{t}\right)^{1 / 2}, \quad k \geq \alpha>0 .
$$

The fractional square function and the fractional $g_{\lambda}^{*}$-function associated to $\left\{P_{t}^{L}\right\}_{t>0}$ are defined, respectively, by

$$
G_{k, \alpha}(f)=\left(\int_{0}^{+\infty} \int_{|x-y|<t}\left|t^{k-\alpha} \frac{\partial^{k} P_{t}^{L} f}{\partial t^{k}}\right|^{2} \frac{d y d t}{t^{d+1}}\right)^{1 / 2}, \quad k \geq \alpha>0
$$

and

$$
g_{k, \alpha ; \lambda}^{*}(f)=\left[\int_{0}^{+\infty} \int_{\mathbf{R}^{d}}\left(\frac{t}{|x-y|+t}\right)^{2 \lambda}\left|t^{k-\alpha} \frac{\partial^{k} P_{t}^{L} f}{\partial t^{k}}\right|^{2} \frac{d y d t}{t^{d+1}}\right]^{1 / 2}, \quad k \geq \alpha>0 .
$$

Define $L_{V}^{p, \alpha}\left(\mathbf{R}^{d}\right)$ as the collection of all functions $f$ such that

$$
f=(I+L)^{-\alpha / 2} g, \quad g \in L^{p}\left(\mathbf{R}^{d}\right),
$$

where $1<p<\infty$. The main result of [5] is the following proposition.

Proposition 1.6. Let $\alpha>0, k \in \mathbf{N} \backslash\{0\}, 1<p<\infty$ and $\lambda>\max \{d / p, d / 2\}$. Then the following assertions are equivalent:

(i) $f \in L_{V}^{p, \alpha}\left(\mathbf{R}^{d}\right)$;

(ii) $f \in L^{p}\left(\mathbf{R}^{d}\right)$ and $g_{k, \alpha}(f) \in L^{p}\left(\mathbf{R}^{d}\right)$ for $k>\alpha$;

(iii) $f \in L^{p}\left(\mathbf{R}^{d}\right)$ and $G_{k, \alpha}(f) \in L^{p}\left(\mathbf{R}^{d}\right)$ for $\alpha<k-(d+1) / 2$; 
(iv) $f \in L^{p}\left(\mathbf{R}^{d}\right)$ and $g_{k, \alpha ; \lambda}^{*}(f) \in L^{p}\left(\mathbf{R}^{d}\right)$ for $\alpha<k-(d+1) / 2$.

Moreover, for every $f \in L_{V}^{p, \alpha}$,

$$
\|f\|_{L_{V}^{p, \alpha}} \sim\|f\|_{L^{p}}+\left\|g_{k, \alpha}(f)\right\|_{L^{p}} \sim\|f\|_{L^{p}}+\left\|G_{k, \alpha}(f)\right\|_{L^{p}} \sim\|f\|_{L^{p}}+\left\|g_{k, \alpha ; \lambda}^{*}(f)\right\|_{L^{p}} .
$$

In this paper, we will give the characterizations of the Hardy-Sobolev space $H_{L}^{1, \alpha}\left(\mathbf{R}^{d}\right)$ as follows.

Theorem 1.7. Let $\alpha \geq \frac{1}{2}, k \in \mathbf{N} \backslash\{0\}$ and $\lambda>d$. Then the following assertions are equivalent:

(i) $f \in H_{L}^{1, \alpha}\left(\mathbf{R}^{d}\right)$;

(ii) $f \in H_{L}^{1}\left(\mathbf{R}^{d}\right)$ and $g_{k, \alpha}(f) \in L^{1}\left(\mathbf{R}^{d}\right)$ for $k>\alpha$;

(iii) $f \in H_{L}^{1}\left(\mathbf{R}^{d}\right)$ and $G_{k, \alpha}(f) \in L^{1}\left(\mathbf{R}^{d}\right)$ for $\alpha<k-(d+1) / 2$;

(iv) $f \in H_{L}^{1}\left(\mathbf{R}^{d}\right)$ and $g_{k, \alpha ; \lambda}^{*}(f) \in L^{1}\left(\mathbf{R}^{d}\right)$ for $\alpha<k-(d+1) / 2$.

Moreover, for every $f \in H_{L}^{1, \alpha}\left(\mathbf{R}^{d}\right)$,

$$
\|f\|_{H_{L}^{1, \alpha}} \sim\|f\|_{H_{L}^{1}}+\left\|g_{k, \alpha}(f)\right\|_{L^{1}} \sim\|f\|_{H_{L}^{1}}+\left\|G_{k, \alpha}(f)\right\|_{L^{1}} \sim\|f\|_{H_{L}^{1}}+\left\|g_{k, \alpha ; \lambda}^{*}(f)\right\|_{L^{1}} .
$$

The paper is organized as follows. In Section 2, we give some estimates of the kernels associated with $e^{-t L}$ and $P_{t}^{L}$. In Section 3, we give the proof of Theorem 1.4. The proof of Theorem 1.7 will be given in Section 4 .

Throughout this article, we will use $c$ and $C$ to denote the positive constants, which are independent of main parameters and may be different at each occurrence. By $B_{1} \sim B_{2}$, we mean that there exists a constant $C>1$ such that $1 / C \leq B_{1} / B_{2} \leq$ $C$.

\section{Preliminaries}

In this section, we give some estimates of the heat kernel and the Poisson kernel associated with $L$ which will be used in the sequel. We first collect some basic facts about the potential $V$ satisfying the reverse Hölder inequality. Obviously, $B_{q_{1}} \subset B_{q_{2}}$ if $q_{1}>q_{2}$. But it is important that the $B_{q}$ class has a property of self improvement; that is, if $V \in B_{q}$, then $V \in B_{q+\varepsilon}$ for some $\varepsilon>0$. We have assumed that $V \in B_{d / 2}$, and hence $V \in B_{q_{0}}$ for some $q_{0}>d / 2$. We also write $\delta_{0}=2-d / q_{0}>0$ and $\delta=\min \left(1, \delta_{0}\right) \leq 1$, and throughout the paper we keep this assumption and the meanings of $q_{0}, \delta_{0}$ and $\delta$.

We first give the following estimates of the kernels $K_{t}^{L}(\cdot, \cdot)$ (cf. [17]).

Proposition 2.1. (a) For every $N \in \mathbf{N}$, there is a constant $C_{N}>0$ such that

$$
0 \leq K_{t}^{L}(x, y) \leq C_{N} t^{-d / 2} e^{-|x-y|^{2} / 5 t}(1+\sqrt{t} / \rho(x)+\sqrt{t} / \rho(y))^{-N} .
$$

(b) There exist $\delta>0$ and $C>0$ such that for every $N>0$, there is a constant $C_{N}>0$ so that, for all $|h| \leq \sqrt{t}$,

$$
\begin{aligned}
& \left|K_{t}^{L}(x+h, y)-K_{t}^{L}(x, y)\right| \\
& \leq C_{N}(|h| / \sqrt{t})^{\delta} t^{-d / 2} e^{-A|x-y|^{2} / t}(1+\sqrt{t} / \rho(x)+\sqrt{t} / \rho(y))^{-N} .
\end{aligned}
$$

Remark 2.2. By part (a) of Proposition 2.1, it is easy to see that the condition $|h| \leq \sqrt{t}$ can be replaced by $|h| \leq|x-y| / 2$ in part (b) of Proposition 2.1.

For $k \in \mathbf{N}$, define

$$
Q_{t}^{k}(x, y):=\left.t^{2 k} \partial_{s}^{k} K_{s}^{L}(x, y)\right|_{s=t^{2}}
$$


The following estimates about the kernel $Q_{t}^{k}(\cdot, \cdot)$ is obtained in [15].

Proposition 2.3. There exist constants $C, 0<\delta^{\prime}<\delta$ such that for every $N$, there exist constants $C_{N}>0$ and $C_{k, N}>0$, so that

(a) $\left|Q_{t}^{k}(x, y)\right| \leq C_{N} t^{-d} e^{-C|x-y|^{2} / t^{2}}(1+t / \rho(x)+t / \rho(y))^{-N} ;$

(b) $\left|Q_{t}^{k}(x+h, y)-Q_{t}^{k}(x, y)\right| \leq C_{k, N}\left(\frac{|h|}{t}\right)^{\delta^{\prime}} t^{-d} e^{-C|x-y|^{2} / t^{2}}(1+t / \rho(x)+t / \rho(y))^{-N}$, $|h| \leq t$

(c) $\left|\int_{\mathbf{R}^{d}} Q_{t}^{k}(x, y) d y\right| \leq C_{N} \frac{(t / \rho(x))^{\delta^{\prime}}}{(1+t / \rho(x))^{N}}$.

By the Bochner's subordination formula, for any $x \in \mathbf{R}^{d}$ and $t>0$,

$$
P_{t}^{L} f(x)=e^{-t \sqrt{L}} f(x)=\frac{1}{\sqrt{\pi}} \int_{0}^{\infty} \frac{e^{-u}}{\sqrt{u}} T_{t^{2} /(4 u)}^{L} f(x) d u=\frac{t}{2 \sqrt{\pi}} \int_{0}^{\infty} \frac{e^{-t^{2} /(4 u)}}{u^{3 / 2}} T_{u}^{L} f(x) d u .
$$

The above identity indicates that the Poisson kernel is given by

$$
P_{t}^{L}(x, y)=\frac{1}{\sqrt{\pi}} \int_{0}^{\infty} \frac{e^{-u}}{\sqrt{u}} K_{t^{2} /(4 u)}^{L}(x, y) d u=\frac{t}{2 \sqrt{\pi}} \int_{0}^{\infty} \frac{e^{-t^{2} /(4 u)}}{u^{3 / 2}} K_{u}^{L}(x, y) d u .
$$

The kernel $\partial_{t}^{\alpha} P_{t}^{L}(\cdot, \cdot)$ satisfies the following estimates.

Proposition 2.4. [Proposition 3.6] Let $\alpha>0$. For any $N>0$ and $0<\delta^{\prime} \leq \delta$ with $0<\delta^{\prime}<\alpha$, there exists a constant $C_{N}$ such that for any $x, y \in \mathbf{R}^{d}$ and $t \in(0, \infty)$,

(a) $0 \leq P_{t}^{L}(x, y) \leq \frac{C_{N} t}{\left(t^{2}+|x-y|^{2}\right)^{(d+1) / 2}}(1+t / \rho(x)+t / \rho(y))^{-N} ;$

(b) $\left|t^{\alpha} \partial_{t}^{\alpha} P_{t}^{L}(x, y)\right| \leq \frac{C_{N} t^{\alpha}}{\left(t^{2}+|x-y|^{2}\right)^{(d+\alpha) / 2}}(1+t / \rho(x)+t / \rho(y))^{-N}$;

(c) $\left|t^{\alpha} \partial_{t}^{\alpha} P_{t}^{L}(x+h, y)-t^{\alpha} \partial_{t}^{\alpha} P_{t}^{L}(x, y)\right| \leq \frac{C_{N} t^{\alpha}(|h| / t)^{\delta^{\prime}}}{\left(t^{2}+|x-y|^{2}\right)^{(d+\alpha) / 2}}(1+t / \rho(x)+t / \rho(y))^{-N}$, $|h| \leq t$

(d) $\left|\int_{0}^{\infty} t^{\alpha} \partial_{t}^{\alpha} P_{t}^{L}(x, y) d y\right| \leq C_{N} \frac{(t / \rho(x))^{\delta^{\prime}}}{(1+t / \rho(x))^{N}}$.

We can express $\partial_{t}^{\alpha} P_{t}^{L} f$ as follows (cf. [37]).

Lemma 2.5. Let $\alpha>0$. For every $f \in L^{2}\left(\mathbf{R}^{d}\right)$,

$$
\partial_{t}^{\alpha} P_{t}^{L} f=e^{i \pi \alpha} \int_{0}^{\infty} \lambda^{\alpha / 2} e^{-t \sqrt{\lambda}} d E_{L}(\lambda) f, \quad t>0 .
$$

At the end of this section, we give some basic facts on the tent spaces which will be used in the sequel(cf. [10]). Let $0<p<\infty$, and $1 \leq q \leq \infty$. The tent space $T_{q}^{p}$ is defined as the space of all functions $u(\cdot, \cdot)$ on $\mathbf{R}_{+}^{d+1}$ such that

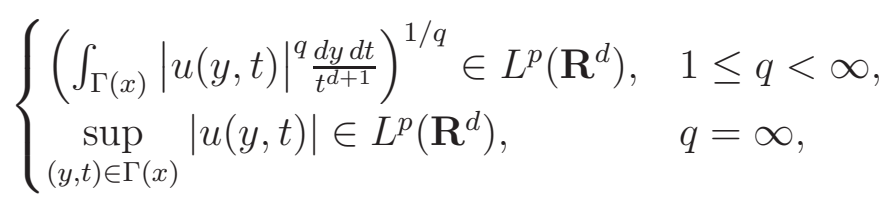

where $\Gamma(x)$ is the standard cone whose vertex is $x \in \mathbf{R}^{d}$, i.e.,

$$
\Gamma(x)=\{(y, t):|y-x|<t\} .
$$


Assume $B\left(x_{0}, r\right)$ is a ball in $\mathbf{R}^{d}$, its tent $\widehat{B}$ is defined by $\widehat{B}=\left\{(x, t):\left|x-x_{0}\right| \leq r-t\right\}$. A function $a(\cdot, \cdot)$ that supported in a tent $\widehat{B}$, where $B$ is a ball in $\mathbf{R}^{d}$, is said to be an atom in the tent space $T_{2}^{p}$ if it satisfies

$$
\left(\int_{\widehat{B}}|a(x, t)|^{2} \frac{d x d t}{t}\right)^{1 / 2} \leq|B|^{1 / 2-1 / p}
$$

The atomic decomposition of $T_{2}^{p}$ is stated as follows (cf. [10]).

Proposition 2.6. Let $0<p \leq 1$. Any $u \in T_{2}^{p}$ can be written as $u=\sum \lambda_{k} a_{k}$, where $\left\{a_{k}\right\}$ are atoms and $\sum\left|\lambda_{k}\right|^{p} \leq C\|u\|_{T_{2}^{p}}^{p}$.

\section{The characterization of the Hardy space $H_{L}^{1}\left(\mathrm{R}^{d}\right)$}

In this section, we will give the characterizations of Hardy space $H_{L}^{1}\left(\mathbf{R}^{d}\right)$ by the fractional square functions. Firstly, we give the following lemmas, which will be used in the sequel (cf. [15] or [31]).

Lemma 3.1. Let $\alpha>0$. The operator $t^{\alpha} \partial_{t}^{\alpha} P_{t}^{L}$ defines an isometry from $L^{2}\left(\mathbf{R}^{d}\right)$ into $L^{2}\left(\mathbf{R}_{+}^{d+1}, d x d t / t\right)$. Moreover,

$$
f(x)=\frac{4^{\alpha}}{\Gamma(2 \alpha)} \lim _{\epsilon \rightarrow 0} \lim _{N \rightarrow \infty} \int_{\epsilon}^{N}\left(t^{\alpha} \partial_{t}^{\alpha} P_{t}^{L}\right)^{2} f(x) \frac{d t}{t} \quad \text { in } L^{2}\left(\mathbf{R}^{d}\right) .
$$

By the spectral theorem, we can prove

Lemma 3.2. Let $\alpha>0$. The operators $g_{\alpha}, G_{\alpha}$ and $g_{\alpha, \lambda}^{*}$ are bounded on $L^{2}\left(\mathbf{R}^{d}\right)$. Moreover, there exist constants $C, C_{1}$ and $C_{2}$ such that

$$
\left\|g_{\alpha} f\right\|_{L^{2}}=C\|f\|_{L^{2}}, \quad\left\|G_{\alpha} f\right\|_{L^{2}} \leq C_{1}\|f\|_{L^{2}}, \quad\left\|g_{\alpha, \lambda}^{*} f\right\|_{L^{2}} \leq C_{2}\|f\|_{L^{2}} .
$$

The following proposition has been proved in [31].

Proposition 3.3. [31, Lemma 5.3] Let $\alpha>0$. There exists a constant $C$ such that for any function $f$ which is a linear combination of $H_{L}^{1}$-atoms, we have $\left\|G_{\alpha} f\right\|_{L^{1}} \leq C\|f\|_{H_{L}^{1}}$.

Similar to the proof of Proposition 3.3, we can prove

Corollary 3.4. Let $\alpha>0$. There exists a constant $C$ such that for any function $f$ which is a linear combination of $H_{L^{1}}^{1}$ atoms, we have $\left\|g_{\alpha} f\right\|_{L^{1}} \leq C\|f\|_{H_{L}^{1}}$ and $\left\|g_{\alpha, \lambda}^{*} f\right\|_{L^{1}} \leq C\|f\|_{H_{L}^{1}}$.

In order to prove the main result, we need the following Lemma (cf. [21]).

Lemma 3.5. $f \in H_{L}^{1}\left(\mathbf{R}^{d}\right)$ if and only if $f \in L^{1}\left(\mathbf{R}^{d}\right)$ and $M_{P}(f)=\sup _{s>0} P_{s}^{L}(f) \in$ $L^{1}\left(\mathbf{R}^{d}\right)$. Moreover, we have

$$
\|f\|_{H_{L}^{1}} \sim\left\|M_{P}(f)\right\|_{L^{1}}
$$

Now, we can give the proof of Theorem 1.4.

Proof of Theorem 1.4. By Proposition 3.3 and Corollary 3.4, for $f \in H_{L}^{1}\left(\mathbf{R}^{d}\right)$, we know that $g_{\alpha} f \in L^{1}\left(\mathbf{R}^{d}\right), G_{\alpha} f \in L^{1}\left(\mathbf{R}^{d}\right)$ and $g_{\alpha, \lambda}^{*} f \in L^{1}\left(\mathbf{R}^{d}\right)$, respectively.

For the reverse, we first show that for $G_{\alpha} f \in L^{1}\left(\mathbf{R}^{d}\right)$, we have $f \in H_{L}^{1}\left(\mathbf{R}^{d}\right)$. In the following, for the sake of convenience, we use $D_{t}^{\alpha}(\cdot, \cdot)$ to denote the kernel $t^{\alpha} \partial_{t}^{\alpha} P_{t}(\cdot, \cdot)$. Assume that $f \in L^{1}\left(\mathbf{R}^{d}\right) \cap L^{2}\left(\mathbf{R}^{d}\right)$. When $G_{k, \alpha} f \in L^{1}\left(\mathbf{R}^{d}\right)$, we can see that

$$
\int_{\mathbf{R}^{d}}\left|G_{\alpha} f(x)\right| d x=\int_{\mathbf{R}^{d}}\left(\int_{0}^{\infty} \int_{B(x, t)}\left|D_{t}^{\alpha} f(y)\right|^{2} \frac{d y d t}{t^{d+1}}\right)^{1 / 2} d x,
$$


which implies that $D_{t}^{\alpha} f(x)$ belongs to the tent space $T_{2}^{1}$, where

$$
D_{t}^{\alpha} f(x):=\int_{\mathbf{R}^{d}} D_{t}^{\alpha}(x, y) f(y) d y
$$

By the atomic decomposition of $T_{2}^{1}$, we have $D_{t}^{\alpha} f(x)=\sum_{i} \lambda_{i} a_{i}(x, t)$, where $a_{i}(x, t)$ are $T_{2}^{1}$-atoms and $\sum_{i}\left|\lambda_{i}\right|<\infty$. Assume that the atom $a(x, t)$ is supported on $\widehat{B}\left(x_{0}, r\right)$. By Lemma 3.1,

$$
f(x)=C \int_{0}^{\infty} D_{t}^{\alpha}\left(\sum_{i=1}^{\infty} \lambda_{i} a_{i}(x, t)\right) \frac{d t}{t}:=\sum_{i=1}^{\infty} \lambda_{i} \tau_{i}(x),
$$

where $\tau_{i}(x)=\int_{0}^{\infty} D_{t}^{\alpha} a_{i}(x, t) \frac{d t}{t}$. For simplicity, we use $\tau(x)$ to denote $\tau_{i}(x)$ for $i=$ $1,2, \cdots$. We write

$$
\begin{aligned}
\left\|\sup _{t>0}\left|e^{-t \sqrt{L}} \tau(x)\right|\right\|_{L^{1}} & \leq\left\|\left(\sup _{t>0}\left|e^{-t \sqrt{L}} \tau(x)\right|\right) \chi_{B^{*}}\right\|_{L^{1}}+\left\|\left(\sup _{t>0}\left|e^{-t \sqrt{L}} \tau(x)\right|\right) \chi_{\left(B^{*}\right)^{c}}\right\|_{L^{1}} \\
& :=I_{1}+I_{2},
\end{aligned}
$$

where $B^{*}=B\left(x_{0}, 2 r\right)$. For $I_{1}$, we use Hölder's inequality to deduce that

$$
\begin{aligned}
\|\tau\|_{2} & =\sup _{\|\phi\|_{2} \leq 1} \int_{\mathbf{R}^{d}}\left(\int_{0}^{\infty} D_{t}^{\alpha} a(x, t) \frac{d t}{t}\right) \bar{\phi}(x) d x \\
& \leq \sup _{\|\phi\|_{2} \leq 1}\left(\int_{0}^{\infty} \int_{\mathbf{R}^{d}}|a(x, t)|^{2} \frac{d t d x}{t}\right)^{1 / 2}\left(\int_{0}^{\infty} \int_{\mathbf{R}^{d}}\left|D_{t}^{\alpha} \bar{\phi}(x)\right|^{2} \frac{d t d x}{t}\right)^{1 / 2} \\
& \leq \sup _{\|\phi\|_{2} \leq 1}|B|^{-1 / 2}\|\phi\|_{2} \leq|B|^{-1 / 2}
\end{aligned}
$$

which gives $I_{1} \leq\left|B^{*}\right|^{1 / 2}|B|^{-1 / 2} \leq C$.

Now we deal with $I_{2}$. For $s>0$, by functional calculus and Proposition 2.4, we have

$$
\begin{aligned}
\left|e^{-s \sqrt{L}}\left(\int_{0}^{\infty} D_{t}^{\alpha} a(x, t) \frac{d t}{t}\right)\right| & =C\left|e^{-s \sqrt{L}} \int_{0}^{\infty} \int_{0}^{\infty} t^{\alpha} \partial_{t} P_{t+\lambda}^{L} a(x, t) r^{1-\alpha} \frac{d \lambda}{\lambda} \frac{d t}{t}\right| \\
& =\left|\int_{0}^{\infty} \int_{0}^{\infty} t^{\alpha} \partial_{t} P_{s+t+\lambda}^{L} a(x, t) \lambda^{1-\alpha} \frac{d \lambda}{\lambda} \frac{d t}{t}\right| \\
& =\left|\int_{0}^{\infty} t^{\alpha} \partial_{t}^{\alpha} P_{s+t}^{L} a(x, t) \frac{d t}{t}\right| \\
& \leq C \int_{0}^{\infty} \frac{t^{\alpha}}{\left((s+t)^{2}+|x-y|^{2}\right)^{\frac{d+\alpha}{2}}}|a(y, t)| \frac{d y d t}{t} .
\end{aligned}
$$

When $y \in B\left(x_{0}, r\right)$ and $x \in\left(B^{*}\right)^{c}$, we have $|x-y| \sim\left|x-x_{0}\right|$. Then

$$
\begin{aligned}
& \left|e^{-s \sqrt{L}}\left(\int_{0}^{\infty} D_{t}^{\alpha} a(x, t) \frac{d t}{t}\right)\right| \\
& \leq C\left|x-x_{0}\right|^{-(d+\alpha)}\left(\int_{0}^{r} \int_{B} t^{2 \alpha-1} d y d t\right)^{1 / 2}\left(\int_{0}^{r} \int_{B}|a(y, t)|^{2} \frac{d y d t}{t}\right)^{1 / 2} \\
& \leq C|B|^{-\frac{1}{2}}\left|x-x_{0}\right|^{-(d+\alpha)}\left(\int_{0}^{r} \int_{B} t^{2 \alpha-1} d y d t\right)^{1 / 2} \leq C r^{\alpha}\left|x-x_{0}\right|^{-(d+\alpha)} .
\end{aligned}
$$

Finally, we get

$$
I_{2} \leq \int_{B^{c}\left(x_{0}, r\right)} \frac{r^{\alpha}}{\left|x-x_{0}\right|^{d+\alpha}} d x \leq C .
$$


When $f \in H_{L}^{1}\left(\mathbf{R}^{d}\right)$, let $\widetilde{G_{\alpha}}$ be the bounded extension of $G_{\alpha}(f)$ from $L^{2} \cap H_{L}^{1}\left(\mathbf{R}^{d}\right)$ to $H_{L}^{1}\left(\mathbf{R}^{d}\right)$. Since $L^{2} \cap H_{L}^{1}\left(\mathbf{R}^{d}\right)$ is dense in $H_{L}^{1}\left(\mathbf{R}^{d}\right)$, there exists a sequence $\left\{f_{n}\right\} \subset$ $L^{2} \cap H_{L}^{1}\left(\mathbf{R}^{d}\right)$ such that $f_{n} \rightarrow f$ as $n \rightarrow \infty$ in $H_{L}^{1}\left(\mathbf{R}^{d}\right)$. By Proposition 2.4, we conclude that $G_{\alpha}\left(f_{n}\right) \rightarrow G_{\alpha}(f)$ as $n \rightarrow \infty$. By the definition of $\widetilde{G_{\alpha}}$, we know that $G_{\alpha}\left(f_{n}\right) \rightarrow \widetilde{G_{\alpha}}(f)$ as $n \rightarrow \infty$. Therefore, for $f \in H_{L}^{1}\left(\mathbf{R}^{d}\right), G_{\alpha}(f)=\widetilde{G_{\alpha}}(f)$. This gives

$$
\|f\|_{H_{L}^{1}}=\left\|\lim _{n \rightarrow \infty} f_{n}\right\|_{H_{L}^{1}} \leq \lim _{n \rightarrow \infty}\left\|G_{\alpha}\left(f_{n}\right)\right\|_{L^{1}}=\left\|\widetilde{G_{\alpha}}(f)\right\|_{L^{1}}=\left\|G_{\alpha}(f)\right\|_{L^{1}} .
$$

For the Littlewood-Paley $g$-function, it is sufficient to prove $\left\|G_{\alpha}(f)\right\|_{L^{1}} \leq C\left\|g_{\alpha}(f)\right\|_{L^{1}}$. For $\beta>0$, we define $\widetilde{G_{\beta}}(f)$ by

$$
\widetilde{G_{\beta}}(f)(x)=\left(\int_{0}^{+\infty} \int_{|x-y|<\beta t}\left|D_{t}^{\alpha} f(y)\right|^{2} \frac{d y d t}{t^{d+1}}\right)^{1 / 2} .
$$

Then same as the above proof, we can prove that $f \in H_{L}^{1}\left(\mathbf{R}^{d}\right)$ if and only if $\widetilde{G_{\beta}}(f) \in$ $L^{1}\left(\mathbf{R}^{d}\right)$ and $f \in L^{1}\left(\mathbf{R}^{d}\right)$. Moreover, $\|f\|_{H_{L}^{1}} \sim\left\|\widetilde{G_{\beta}}(f)\right\|_{L^{1}}$.

Let $F(x)(t):=\left(\partial_{t}^{\alpha} e^{-t \sqrt{L}} f\right)(x)$ and $V(x, s):=e^{-s \sqrt{L}} F(x)$. Then

$$
V(x, s)(t)=e^{-s \sqrt{L}}\left(\partial_{t}^{\alpha} e^{-t \sqrt{L}} f\right)(x)=\left(\partial_{t}^{\alpha} e^{-(s+t) \sqrt{L}} f\right)(x) .
$$

Therefore,

$$
\begin{aligned}
\int_{0}^{+\infty}|V(x, s)(t)|^{2} t^{2 \alpha-1} d t & =\int_{0}^{+\infty}\left|\left(\partial_{t}^{\alpha} e^{-(s+t) \sqrt{L}} f\right)(x)\right|^{2} t^{2 \alpha-1} d t \\
& =\int_{s}^{+\infty}\left|\left(\partial_{t}^{\alpha} e^{-t \sqrt{L}} f\right)(x)\right|^{2}(t-s)^{2 \alpha-1} d t
\end{aligned}
$$

When $\alpha \geq \frac{1}{2}$, we have $(t-s)^{2 \alpha-1} \leq t^{2 \alpha-1}$. Hence,

$$
\sup _{s>0} \int_{0}^{+\infty}|V(x, s)(t)|^{2} t^{2 \alpha-1} d t \leq \int_{0}^{+\infty}\left|\left(\partial_{t}^{\alpha} e^{-t \sqrt{L}} f\right)(x)\right|^{2} t^{2 \alpha-1} d t=\left(g_{\alpha} f(x)\right)^{2} .
$$

Let $\mathbf{X}=L^{2}\left((0, \infty), t^{2 \alpha-1} d t\right)$. Then

$$
\sup _{s>0}\left\|e^{-s L} F(x)\right\|_{\mathbf{X}} \leq g_{\alpha} f(x) \in L^{1}\left(\mathbf{R}^{d}\right) .
$$

Therefore, $F \in H_{\mathbf{X}}^{1}\left(\mathbf{R}^{d}\right)$, where $H_{\mathbf{X}}^{1}\left(\mathbf{R}^{d}\right)$ can be seen as a vector-valued Hardy space (cf. [19]). This shows that $\tilde{G}_{2}^{\mathbf{X}} F(x) \in L^{1}\left(\mathbf{R}^{d}\right)$, where

$$
\tilde{G}_{2}^{\mathbf{X}} F(x)=\left(\int_{0}^{+\infty} \int_{|x-y|<2 t}\left\|D_{t}^{\alpha} F(y)\right\|_{\mathbf{X}}^{2} \frac{d y d t}{t^{d+1}}\right)^{1 / 2} .
$$

We can assume that $\frac{1}{2} \leq \alpha<1$. Then the identity

$$
\partial_{t}^{\alpha} P_{t}^{L}=C \int_{0}^{\infty} \partial_{s} P_{s+t}^{L} s^{-\alpha} d s
$$


gives

$$
\begin{aligned}
\partial_{t}^{\alpha} P_{t}^{L} \partial_{s}^{\alpha} P_{s}^{L} & =C \int_{0}^{\infty} \int_{0}^{\infty} \partial_{a} P_{a+t}^{L} \partial_{b} P_{s+b}^{L} a^{-\alpha} d a b^{-\alpha} d b \\
& =C \int_{0}^{\infty} \int_{0}^{\infty} \partial_{a}^{2} P_{a+b+s+t}^{L} a^{-\alpha} b^{-\alpha} d a d b \\
& =C \int_{0}^{\infty} \int_{a}^{\infty} \partial_{\lambda}^{2} P_{\lambda+s+t}^{L} a^{-\alpha}(\lambda-a)^{-\alpha} d \lambda d a \\
& =C \int_{0}^{\infty} \partial_{\lambda}^{2} P_{\lambda+s+t}^{L}\left(\int_{0}^{\lambda} a^{-\alpha}(\lambda-a)^{-\alpha} d a\right) d \lambda \\
& =C \int_{0}^{\infty} \partial_{\lambda}^{2} P_{\lambda+s+t}^{L}\left[\left(\int_{0}^{\lambda / 2}+\int_{\lambda / 2}^{\lambda}\right) a^{-\alpha}(\lambda-a)^{-\alpha} d a\right] d \lambda \\
& =C \int_{0}^{\infty} \partial_{\lambda}^{2} P_{\lambda+s+t}^{L} \lambda^{1-2 \alpha} d \lambda .
\end{aligned}
$$

When $\alpha \geq 1 / 2$, we get

$$
\partial_{t}^{\alpha} P_{t}^{L} \partial_{s}^{\alpha} P_{s}^{L}=\partial_{t}^{2 \alpha} P_{s+t}^{L}
$$

Then

$$
\begin{aligned}
& \left(\tilde{G}_{2}^{\mathbf{X}} F(x)\right)^{2}=\int_{0}^{+\infty} \int_{|x-y|<2 t}\left\|D_{t}^{\alpha} F(y)\right\|_{\mathbf{X}}^{2} \frac{d y d t}{t^{d+1}} \\
& =\int_{0}^{+\infty} \int_{|x-y|<2 t} \int_{0}^{+\infty}\left|t^{\alpha} \partial_{t}^{2 \alpha} e^{-t \sqrt{L}} F(y)(s)\right|^{2} s^{2 \alpha-1} d s \frac{d y d t}{t^{d+1}} \\
& =\int_{0}^{+\infty} \int_{0}^{+\infty} \int_{|x-y|<2 t}\left|\partial_{t}^{2 \alpha} e^{-(s+t) \sqrt{L}} f(y)\right|^{2} t^{2 \alpha-1-d} s^{2 \alpha-1} d y d t d s \\
& =\int_{0}^{+\infty} \int_{s}^{+\infty} \int_{|x-y|<2(t-s)}\left|\partial_{t}^{2 \alpha} e^{-t \sqrt{L}} f(y)\right|^{2}(t-s)^{2 \alpha-1-d} s^{2 \alpha-1} d y d t d s \\
& =\int_{0}^{+\infty} \int_{0}^{t} \int_{|x-y|<2(t-s)}\left|\partial_{t}^{2 \alpha} e^{-t \sqrt{L}} f(y)\right|^{2}(t-s)^{2 \alpha-1-d} s^{2 \alpha-1} d y d s d t \\
& \geq \int_{0}^{+\infty} \int_{0}^{t / 2} \int_{|x-y|<2(t-s)}\left|\partial_{t}^{2 \alpha} e^{-t \sqrt{L}} f(y)\right|^{2}(t-s)^{2 \alpha-1-d} s^{2 \alpha-1} d y d s d t \\
& \geq \int_{0}^{+\infty} \int_{0}^{t / 2} \int_{|x-y|<t}\left|\partial_{t}^{2 \alpha} e^{-t \sqrt{L}} f(y)\right|^{2} t^{2 \alpha-1-d} s^{2 \alpha-1} d y d s d t \\
& =\frac{2 \alpha}{8} \int_{0}^{+\infty} \int_{|x-y|<t}\left|\partial_{t}^{2 \alpha} e^{-t \sqrt{L}} f(y)\right|^{2} t^{4 \alpha-1-d} d y d t \\
& =\frac{2 \alpha}{8} \int_{0}^{+\infty} \int_{|x-y|<t}\left|t^{2 \alpha} \partial_{t}^{2 \alpha} e^{-t \sqrt{L}} f(y)\right|^{2} \frac{d y d t}{t^{d+1}}=\frac{2 \alpha}{8}\left(\widetilde{S_{L}^{1}} f(x)\right)^{2},
\end{aligned}
$$

which implies $G_{\alpha}(f) \in L^{1}\left(\mathbf{R}^{d}\right)$. Hence, $f \in H_{L}^{1}\left(\mathbf{R}^{d}\right)$.

Since in the cone $\Gamma(x)=\{(y, t):|x-y|<t\}$, we have $[t /(|x-y|+t)]^{2 \lambda}>2^{-2 \lambda}$. Therefore,

$$
G_{\alpha}(f)(x) \leq\left[\int_{\Gamma(x)} 2^{2 \lambda}\left(\frac{t}{|x-y|+t}\right)^{2 \lambda}\left|t^{\alpha} \partial_{t}^{\alpha} P_{t}^{L} f(y)\right|^{2} \frac{d y d t}{t^{d+1}}\right]^{1 / 2} \leq 2^{\lambda} G_{\alpha, \lambda}^{*}(f)(x)
$$

This completes the proof of Theorem 1.4. 


\section{The characterization of the Hardy-Sobolev space $H_{L}^{1, \alpha}\left(\mathrm{R}^{d}\right)$}

We denote by $E_{L}$ the spectral decomposition of the operator $L$. If $M$ is a bounded function on $(0, \infty)$, the spectral multiplier $M(L)$ is defined by

$$
M(L) f=\int_{0}^{\infty} M(\lambda) d E_{L}(\lambda) f, \quad f \in D(M(L)),
$$

where the domain

$$
D(M(L))=\left\{f \in L^{2}\left(\mathbf{R}^{d}\right): \int_{0}^{\infty}|M(\lambda)|^{2}\left\langle d E_{L}(\lambda) f, f\right\rangle<\infty\right\} .
$$

We say that a function $M$ on $\mathbf{R}$ belongs to the space $C(s), s \geq 0$, if

$$
\begin{cases}\|M\|_{C(s)}:=\sum_{k=0}^{s} \sup \left|M^{(k)}(\lambda)\right|<\infty, & s \in \mathbf{Z} ; \\ \|M\|_{C(s)}:=\left\|M^{([s])}\right\|_{L i p(s-[s])}+\sum_{k=0}^{[s]} \sup \left|M^{(k)}(\lambda)\right|<\infty, & s \in \mathbf{Z}^{c} .\end{cases}
$$

We have the following version of spectral multiplier theorems.

Proposition 4.1. [14, Theorem 1.11] Let $M$ be a bounded continuous function on $(0, \infty)$. If for some $\epsilon>0$ and a nonzero function $\phi \in C_{c}^{\infty}(0, \infty)$, there exists a constant $C>0$ such that for every $t>0$,

$$
\|\phi(\cdot) M(t \cdot)\|_{C(d / 2+\epsilon)} \leq C,
$$

then the operator $M(L)$ is bounded on $H_{L}^{1}\left(\mathbf{R}^{d}\right)$.

For $\alpha, \beta>0$, let

$$
M_{1}(\lambda)=\frac{\lambda^{\alpha}}{(1+\lambda)^{\alpha}}, \quad M_{2}(\lambda)=\frac{(1+\lambda)^{\alpha}}{1+\lambda^{\alpha}}, \quad M_{3}(\lambda)=(\beta+\lambda)^{-\alpha},
$$

where $\lambda>0$. Then, it is clear that $M_{j}, j=1,2,3$, are smooth and bounded on $(0, \infty)$. It follows from Proposition 4.1 that

Proposition 4.2. Let $\alpha, \beta>0$. Then, for $j=1,2,3$, the operators $M_{j}(L)$ can be extended to bounded operators on $H_{L}^{1}\left(\mathbf{R}^{d}\right)$.

By Lemma 2.5, we can get

$$
\partial_{t}^{k-\alpha} P_{t}^{L}\left(L^{\alpha / 2} f\right)=L^{(k-\alpha) / 2} P_{t}^{L}\left(L^{\alpha / 2} f\right)=L^{k / 2} P_{t}^{L}(f)=\partial_{t}^{k} P_{t}^{L}(f) .
$$

Therefore, we can prove (cf. [5] Proposition 4.1)

Lemma 4.3. Let $0<\alpha<k, k \in \mathbf{N}$ and $\lambda>d / 2$. For every $f \in D\left(L^{\alpha / 2}\right)$, we have

$$
g_{k-\alpha}\left(L^{\alpha / 2} f\right)=g_{k, \alpha}(f), \quad G_{k-\alpha}\left(L^{\alpha / 2} f\right)=G_{k, \alpha}(f), \quad g_{k-\alpha, \lambda}^{*}\left(L^{\alpha / 2} f\right)=g_{k, \alpha, \lambda}^{*}(f) .
$$

By Lemma 4.3 and Theorem 1.4, we get

Proposition 4.4. Let $0<\alpha<k, k \in \mathbf{N}$ and $\lambda>d$. Suppose that

$$
f \in D\left(L^{\alpha / 2}\right) \cap H_{L}^{1}\left(\mathbf{R}^{d}\right) \quad \text { and } \quad L^{\alpha / 2} f \in L^{2}\left(\mathbf{R}^{d}\right) \cap H_{L}^{1}\left(\mathbf{R}^{d}\right) .
$$

Then

$$
\left\|L^{\alpha / 2} f\right\|_{H_{L}^{1}} \sim\left\|g_{k, \alpha}(f)\right\|_{L^{1}} \sim\left\|G_{k, \alpha}(f)\right\|_{L^{1}} \sim\left\|g_{k, \alpha, \lambda}^{*}(f)\right\|_{L^{1}} .
$$

Now, we can prove 
Theorem 4.5. Let $0<\alpha<k, k \in \mathbf{N}$ and $\lambda>d$. Suppose that

$$
f \in D\left((I+L)^{\alpha / 2}\right) \cap H_{L}^{1}\left(\mathbf{R}^{d}\right) \text { and }(I+L)^{\alpha / 2} f \in L^{2}\left(\mathbf{R}^{d}\right) \cap H_{L}^{1}\left(\mathbf{R}^{d}\right) .
$$

Then

$$
\begin{aligned}
\left\|(I+L)^{\alpha / 2} f\right\|_{H_{L}^{1}} & \sim\|f\|_{H_{L}^{1}}+\left\|g_{k, \alpha}(f)\right\|_{L^{1}} \sim\|f\|_{H_{L}^{1}}+\left\|G_{k, \alpha}(f)\right\|_{L^{1}} \\
& \sim\|f\|_{H_{L}^{1}}+\left\|g_{k, \alpha, \lambda}^{*}(f)\right\|_{L^{1}} .
\end{aligned}
$$

Proof. We give the proof of $\left\|(I+L)^{\alpha / 2} f\right\|_{H_{L}^{1}} \sim\|f\|_{H_{L}^{1}}+\left\|g_{k, \alpha}(f)\right\|_{L^{1}}$. The proofs for the cases of $G_{k, \alpha}(f)$ and $g_{k, \alpha, \lambda}^{*}(f)$ are similar.

By Proposition 4.2, we know that the operators $L^{\alpha / 2}(I+L)^{-\alpha / 2}$ and $(I+L)^{\alpha / 2}(I+$ $\left.L^{\alpha / 2}\right)^{-1}$ are bounded on $H_{L}^{1}\left(\mathbf{R}^{d}\right)$. Then following from Proposition 4.4, we have

$$
\begin{aligned}
\left\|(I+L)^{\alpha / 2} f\right\|_{H_{L}^{1}} & =\left\|(I+L)^{\alpha / 2}\left(I+L^{\alpha / 2}\right)^{-1}\left(I+L^{\alpha / 2}\right) f\right\|_{H_{L}^{1}} \\
& \leq\left\|\left(I+L^{\alpha / 2}\right) f\right\|_{H_{L}^{1}} \leq C\left(\|f\|_{H_{L}^{1}}+\left\|L^{\alpha / 2} f\right\|_{H_{L}^{1}}\right) \\
& \leq C\left[\|f\|_{H_{L}^{1}}+\left\|g_{k, \alpha}(f)\right\|_{H_{L}^{1}}\right] .
\end{aligned}
$$

For the reverse, we take the function $M_{1}(\lambda)=\lambda^{\alpha / 2}(1+\lambda)^{-\alpha / 2}, \lambda>0$. For any $r \in \mathbf{R}^{+}$, we get

$$
\begin{aligned}
\int_{0}^{r} \lambda^{\alpha / 2} d E_{L}(\lambda) f & =\int_{0}^{r} \frac{\lambda^{\alpha / 2}}{(1+\lambda)^{\alpha / 2}}(1+\lambda)^{\alpha / 2} d E_{L}(\lambda) f \\
& =M_{1}(L) \int_{0}^{r}(1+\lambda)^{\alpha / 2} d E_{L}(\lambda) f .
\end{aligned}
$$

Letting $r \rightarrow \infty$, we get $L^{\alpha / 2}(f)=M_{1}(\lambda)(I+L)^{\alpha / 2}(f)$. Using Proposition 4.2 again, we obtain

and

$$
\left\|L^{\alpha / 2} f\right\|_{H_{L}^{1}} \leq C\left\|(I+L)^{\alpha / 2} f\right\|_{H_{L}^{1}}
$$

$$
\|f\|_{H_{L}^{1}}=\left\|(I+L)^{-\alpha / 2}(I+L)^{\alpha / 2} f\right\|_{H_{L}^{1}} \leq C\left\|(I+L)^{\alpha / 2} f\right\|_{H_{L}^{1}} .
$$

Theorem 4.5 follows from Proposition 4.4.

Denote by

$$
S_{\alpha, L}=\left\{f \in H_{L}^{1}\left(\mathbf{R}^{d}\right):(I+L)^{\alpha / 2} f \in C_{c}^{\infty}\left(\mathbf{R}^{d}\right)\right\} .
$$

Since $C_{c}^{\infty}\left(\mathbf{R}^{d}\right)$ is dense in $H_{L}^{1}\left(\mathbf{R}^{d}\right), S_{\alpha, L}$ is dense in $H_{L}^{1, \alpha}\left(\mathbf{R}^{d}\right)$. Note that

$$
S_{\alpha, L} \subset D\left((I+L)^{\alpha / 2}\right) \cap H_{L}^{1}\left(\mathbf{R}^{d}\right)
$$

and

$$
(I+L)^{\alpha / 2} S_{\alpha, L}=C_{c}^{\infty}\left(\mathbf{R}^{d}\right) \subset L^{2}\left(\mathbf{R}^{d}\right) \cap H_{L}^{1}\left(\mathbf{R}^{d}\right) .
$$

By Theorem 4.5, $g_{k, \alpha}, G_{k, \alpha}$ and $g_{k, \alpha, \lambda}^{*}$ can be extended to $H_{L}^{1, \alpha}\left(\mathbf{R}^{d}\right)$ as bounded operators from $H_{L}^{1, \alpha}\left(\mathbf{R}^{d}\right)$ to $L^{1}\left(\mathbf{R}^{d}\right)$. Let $\widetilde{g_{k, \alpha}}$ be the extension of $g_{k, \alpha}$ to $H_{L}^{1, \alpha}\left(\mathbf{R}^{d}\right)$ as a bounded operator from $H_{L}^{1, \alpha}\left(\mathbf{R}^{d}\right)$ to $L^{1}\left(\mathbf{R}^{d}\right)$. Then, there exists $C>0$ such that for $f \in H_{L}^{1, \alpha}\left(\mathbf{R}^{d}\right)$,

$$
\|f\|_{H_{L}^{1}}+\left\|\widetilde{g_{k, \alpha}}(f)\right\|_{1} \leq C\|f\|_{H_{L}^{1, \alpha}}
$$

Now, we give the proof of Theorem 1.7.

Proof of Theorem 1.7. We first prove

$$
\|f\|_{H_{L}^{1}}+\left\|g_{k, \alpha}(f)\right\|_{1} \leq C\|f\|_{H_{L}^{1, \alpha}}
$$


By (4.1), it is sufficient to prove $g_{k, \alpha}(f)=\widetilde{g_{k, \alpha}}(f)$. For $N \in \mathbf{N}$ and $h \in H_{L}^{1}\left(\mathbf{R}^{d}\right)$, by the subordination formula, we have

$$
\left|\frac{\partial^{k}}{\partial t^{k}} P_{t}^{L}(h)(x)\right| \leq C \int_{0}^{\infty} \frac{e^{-t^{2} / 8 u}}{u^{(k+2) / 2}}\left|T_{u}^{L}(h)(x)\right| d u \leq C t^{-k} \sup _{u>0}\left|T_{u}^{L}(h)(x)\right| .
$$

Then

$$
\begin{aligned}
\left(\int_{1 / N}^{\infty}\left|t^{k-\alpha} \frac{\partial^{k}}{\partial t^{k}} P_{t}^{L}(h)(x)\right|^{2} \frac{d t}{t}\right)^{1 / 2} & \leq C\left(\int_{1 / N}^{\infty} t^{-1-2 \alpha} d t\right)^{1 / 2} \sup _{u>0}\left|T_{u}^{L}(h)(x)\right| \\
& \leq C N^{\alpha} \sup _{u>0}\left|T_{u}^{L}(h)(x)\right| .
\end{aligned}
$$

By the definition of $H_{L}^{1}\left(\mathbf{R}^{d}\right)$, we conclude that the operator

$$
h \rightarrow\left(\int_{1 / N}^{\infty}\left|t^{k-\alpha} \frac{\partial^{k}}{\partial t^{k}} P_{t}^{L}(h)(x)\right|^{2} \frac{d t}{t}\right)^{1 / 2}
$$

is bounded from $H_{L}^{1}\left(\mathbf{R}^{d}\right)$ to $L^{1}\left(\mathbf{R}^{d}\right)$.

Therefore, if $f=(I+L)^{-\alpha / 2} h$, where $h \in H_{L}^{1}\left(\mathbf{R}^{d}\right) \cap L^{2}\left(\mathbf{R}^{d}\right)$, we have

$$
\begin{aligned}
& \left\|\left(\int_{1 / N}^{\infty}\left|t^{k-\alpha} \frac{\partial^{k}}{\partial t^{k}} P_{t}^{L}\left((I+L)^{-\frac{\alpha}{2}} h\right)(x)\right|^{2} \frac{d t}{t}\right)^{1 / 2}\right\|_{L^{1}} \\
& =\left\|\left(\int_{1 / N}^{\infty}\left|t^{k-\alpha} \frac{\partial^{k}}{\partial t^{k}} P_{t}^{L}\left(L^{-\frac{\alpha}{2}} M(L) h\right)(x)\right|^{2} \frac{d t}{t}\right)^{1 / 2}\right\|_{L^{1}} \\
& =\left\|\left(\int_{1 / N}^{\infty}\left|t^{k-\alpha} \frac{\partial^{k-\alpha}}{\partial t^{k-\alpha}} P_{t}^{L}(M(L) h)(x)\right|^{2} \frac{d t}{t}\right)^{1 / 2}\right\|_{L^{1}} \\
& \leq C\|M(L) h\|_{H_{L}^{1}} \leq C\|h\|_{H_{L}^{1}},
\end{aligned}
$$

where $C>0$ does not depend on $N \in \mathbf{N}$ and $M(\lambda)=\lambda^{\alpha / 2}(1+\lambda)^{-\alpha / 2}, \lambda>0$. Let $N \rightarrow \infty$, we get

$$
\left\|\widetilde{g_{k, \alpha}}(f)\right\|_{L^{1}}=\left\|\left(\int_{0}^{\infty}\left|t^{k-\alpha} \frac{\partial^{k}}{\partial t^{k}} P_{t}^{L}(f)(x)\right|^{2} \frac{d t}{t}\right)^{1 / 2}\right\|_{L^{1}} \leq C\|f\|_{H_{L}^{1, \alpha}}
$$

By $S_{\alpha, L}$ is dense in $H_{L}^{1, \alpha}\left(\mathbf{R}^{d}\right)$, for $f \in H_{L}^{1, \alpha}\left(\mathbf{R}^{d}\right)$, we have

$$
\left\|g_{k, \alpha}(f)\right\|_{L^{1}}=\left\|\left(\int_{0}^{\infty}\left|t^{k-\alpha} \frac{\partial^{k}}{\partial t^{k}} P_{t}^{L}(f)(x)\right|^{2} \frac{d t}{t}\right)^{1 / 2}\right\|_{L^{1}}=\left\|\widetilde{g_{k, \alpha}}(f)\right\|_{L^{1}} \leq C\|f\|_{H_{L}^{1, \alpha}} .
$$

The proofs for the cases of $G_{k, \alpha}$ and $g_{k, \alpha, \lambda}^{*}$ are similar.

For the reverse, we prove

$$
\left\|\partial_{t}^{\alpha} P_{t}^{L}(f)\right\|_{H_{L}^{1}} \leq C t^{-\alpha}\|f\|_{H_{L}^{1}}
$$


For $m \in \mathbf{N}$ and $m>\alpha$, by (4.2), we have

$$
\begin{aligned}
\left|\sup _{\beta>0} T_{\beta}^{L}\left(\partial_{t}^{\alpha} P_{t}^{L}(f)(x)\right)\right| & \leq\left|\sup _{\beta>0} T_{\beta}^{L}\left(\int_{0}^{\infty} s^{m-\alpha-1} \frac{\partial^{m}}{\partial t^{m}} P_{t+s}^{L}(f)(x) d s\right)\right| \\
& \leq C\left|\sup _{\beta>0} \int_{0}^{\infty} s^{m-\alpha-1} \int_{0}^{\infty} \frac{e^{-(t+s)^{2} / 8 u}}{u^{(m+2) / 2}}\right| T_{u+\beta}^{L}(f)(x) \mid d u d s \\
& \leq C \sup _{u>0}\left|T_{u}^{L}(f)(x)\right| \int_{0}^{\infty} \frac{s^{m-\alpha-1}}{(t+s)^{m}} d s \int_{0}^{\infty} \frac{e^{-1 / v}}{v^{(m+2) / 2}} d v \\
& \leq C t^{-\alpha} \sup _{u>0}\left|T_{u}^{L}(f)(x)\right| .
\end{aligned}
$$

Therefore, (4.3) follows from the definition of $H_{L}^{1}\left(\mathbf{R}^{d}\right)$.

Assume that $f \in H_{L}^{1}\left(\mathbf{R}^{d}\right)$ and $g_{k, \alpha}(f) \in L^{1}\left(\mathbf{R}^{d}\right)$. Let $\left\{f_{n}\right\}$ be a sequence in $C_{c}^{\infty}\left(\mathbf{R}^{d}\right)$ such that $f_{n} \rightarrow f$ as $n \rightarrow \infty$ in $H_{L}^{1}\left(\mathbf{R}^{d}\right)$. Fix $t>0$, denote $f^{t}=P_{t}^{L}(f)$ and $f_{n}^{t}=P_{t}^{L}\left(f_{n}\right), n \in \mathbf{N}$. Then $f^{t}$ and $f_{n}^{t}$ in $H_{L}^{1}\left(\mathbf{R}^{d}\right)$.

By Lemma 2.5 and (4.3), we have

$$
\partial_{t}^{\alpha} f_{n}^{t}=L^{\alpha / 2} f_{n}^{t} \in H_{L}^{1}\left(\mathbf{R}^{d}\right) .
$$

Then, by Proposition 4.1, we get

$$
g_{n}^{t}=M(L)\left(I+L^{\alpha / 2}\right) f_{n}^{t} \in H_{L}^{1}\left(\mathbf{R}^{d}\right),
$$

where $M(\lambda)=(1+\lambda)^{\alpha / 2}\left(1+\lambda^{\alpha / 2}\right)^{-1}, \lambda>0$.

Note that $(I+L)^{-\alpha / 2} g_{n}^{t}=f_{n}^{t}$, we have $f_{n}^{t} \in H_{L}^{1, \alpha}\left(\mathbf{R}^{d}\right)$ and $\left\|f_{n}^{t}\right\|_{H_{L}^{1, \alpha}}=\left\|g_{n}^{t}\right\|_{H_{L}^{1}}$. By (4.3) again,

$$
\partial_{t}^{\alpha} f_{n}^{t} \rightarrow \partial_{t}^{\alpha} f^{t}, \quad \text { as } n \rightarrow \infty \quad \text { in } H_{L}^{1}\left(\mathbf{R}^{d}\right) .
$$

Proposition 4.1 and (4.4) imply that

$$
g_{n}^{t}=M(L)\left(I+L^{\alpha / 2}\right) f_{n}^{t}=M(L)\left(I+\partial_{t}^{\alpha}\right) f_{n}^{t} \rightarrow M(L)\left(I+\partial_{t}^{\alpha}\right) f^{t}
$$

as $n \rightarrow \infty$ in $H_{L}^{1}\left(\mathbf{R}^{d}\right)$. Therefore, there exists $F^{t} \in H_{L}^{1, \alpha}\left(\mathbf{R}^{d}\right)$ such that $f_{n}^{t} \rightarrow F^{t}$ as $n \rightarrow \infty$ in $H_{L}^{1, \alpha}\left(\mathbf{R}^{d}\right)$. So $f_{n}^{t} \rightarrow F^{t}$ as $n \rightarrow \infty$ in $H_{L}^{1}\left(\mathbf{R}^{d}\right)$. Since $f_{n}^{t} \rightarrow f^{t}$ as $n \rightarrow \infty$ in $H_{L}^{1}\left(\mathbf{R}^{d}\right)$, we get $f^{t}=F^{t} \in H_{L}^{1, \alpha}\left(\mathbf{R}^{d}\right)$ and $f_{n}^{t} \rightarrow f^{t}$ as $n \rightarrow \infty$ in $H_{L}^{1, \alpha}\left(\mathbf{R}^{d}\right)$.

Noting that $f_{n}^{t} \in L^{2}\left(\mathbf{R}^{d}\right) \cap H_{L}^{1}\left(\mathbf{R}^{d}\right)$ and $(I+L)^{\alpha / 2} f_{n}^{t} \in L^{2}\left(\mathbf{R}^{d}\right) \cap H_{L}^{1}\left(\mathbf{R}^{d}\right)$, by Theorem 4.5, we get

$$
\left\|f_{n}^{t}\right\|_{H_{L}^{1}}+\left\|g_{k, \alpha}\left(f_{n}^{t}\right)\right\|_{1} \sim\left\|f_{n}^{t}\right\|_{H_{L}^{1, \alpha}}
$$

Letting $n \rightarrow \infty$, we have

$$
\left\|f^{t}\right\|_{H_{L}^{1, \alpha}} \leq C\left(\left\|f^{t}\right\|_{H_{L}^{1}}+\left\|g_{k, \alpha} f^{t}\right\|_{1}\right) .
$$

Since

$$
\begin{aligned}
g_{k, \alpha}\left(f^{t}\right)(x) & =\left(\int_{0}^{\infty}\left|s^{k-\alpha} \frac{\partial^{k}}{\partial s^{k}} P_{s}^{L}\left(f^{t}\right)(x)\right|^{2} \frac{d s}{s}\right)^{1 / 2} \\
& =\int_{0}^{\infty} \mid P_{t}^{L}\left(\left.s^{k-\alpha} \frac{\partial^{k}}{\partial s^{k}} P_{s}^{L}(f)(x)\right|^{2} \frac{d s}{s}\right)^{1 / 2} \\
& \leq P_{t}^{L}\left[\left(\int_{0}^{\infty}\left|s^{k-\alpha} \partial s^{k} P_{s}^{L}(f)(\cdot)\right|^{2} \frac{d s}{s}\right)^{1 / 2}\right](x),
\end{aligned}
$$

we get $\left\|g_{k, \alpha}\left(f^{t}\right)\right\|_{1} \leq\left\|g_{k, \alpha}(f)\right\|_{1}$. Furthermore, this gives

$$
\left\|f^{t}\right\|_{H_{L}^{1, \alpha}} \leq C\left(\left\|f^{t}\right\|_{H_{L}^{1}}+\left\|g_{k, \alpha}\left(f^{t}\right)\right\|_{1}\right) \leq C\left(\|f\|_{H_{L}^{1}}+\left\|g_{k, \alpha}(f)\right\|_{1}\right),
$$


where $C>0$ does not depend on $t$.

By (4.5), we know $\left\{f^{t}\right\}$ are uniformly bounded in $H_{L}^{1, \alpha}\left(\mathbf{R}^{d}\right)$, i.e., $\left\{(I+L)^{\alpha / 2}\left(f^{t}\right)\right\}$ are uniformly bounded in $H_{L}^{1}\left(\mathbf{R}^{d}\right)$. Since $H_{L}^{1}\left(\mathbf{R}^{d}\right)$ is a Banach space, we can find $g \in H_{L}^{1}\left(\mathbf{R}^{d}\right)$ such that

$$
(I+L)^{\alpha / 2}\left(f_{j}^{t}\right) \rightarrow g \quad \text { as } \quad j \rightarrow \infty,
$$

where $\left\{f_{j}^{t}\right\}$ is a subsequence of $\left\{f^{t}\right\}$.

Since $H_{L}^{1}\left(\mathbf{R}^{d}\right)$ is the dual space of $V M O_{L}\left(\mathbf{R}^{d}\right)$ and $C_{c}^{\infty}\left(\mathbf{R}^{d}\right)$ is dense in $V M O_{L}\left(\mathbf{R}^{d}\right)$ with norm of $V M O_{L}\left(\mathbf{R}^{d}\right)$ (cf. [27]), we get

$$
\lim _{j \rightarrow \infty}\left\langle(I+L)^{\alpha / 2}\left(f_{j}^{t}\right), \phi\right\rangle=\langle g, \phi\rangle, \quad \phi \in C_{c}^{\infty}\left(\mathbf{R}^{d}\right) .
$$

By the fact that $(I+L)^{-\alpha / 2}$ is self-adjoint and bounded on $C_{c}^{\infty}\left(\mathbf{R}^{d}\right)$, we have

$$
\lim _{j \rightarrow \infty}\left\langle\left(f_{j}^{t}\right), \phi\right\rangle=\left\langle(I+L)^{-\alpha / 2} g, \phi\right\rangle, \quad \phi \in C_{c}^{\infty}\left(\mathbf{R}^{d}\right) .
$$

Let $h=(I+L)^{-\alpha / 2} g$. Then $h \in H_{L}^{1, \alpha}\left(\mathbf{R}^{d}\right)$ and

$$
\lim _{j \rightarrow \infty}\left\langle\left(f_{j}^{t}\right), \phi\right\rangle=\langle h, \phi\rangle, \quad \phi \in C_{c}^{\infty}\left(\mathbf{R}^{d}\right) .
$$

By the arguments analogous to p.776 in [2], which relay on the decay of the kernel of $P_{t}^{L}$, we can get

$$
\lim _{t \rightarrow 0}\left\langle f^{t}, \phi\right\rangle=\langle f, \phi\rangle, \quad \phi \in C_{c}^{\infty}\left(\mathbf{R}^{d}\right) .
$$

It follows that $f=h$ and

$$
\|f\|_{H_{L}^{1, \alpha}} \leq C\left(\|f\|_{H_{L}^{1}}+\left\|g_{k, \alpha}(f)\right\|_{L^{1}}\right) .
$$

This completes the proof of Theorem 1.7.

Remark 4.6. If we define the following Hardy-Sobolev space $\mathcal{H}_{L}^{1, \alpha}\left(\mathbf{R}^{d}\right)$ as the set of all functions $f \in H_{L}^{1}\left(\mathbf{R}^{d}\right)$ such that $L^{\alpha / 2} f \in H_{L}^{1}\left(\mathbf{R}^{d}\right)$, with the norm

$$
\|f\|_{\mathcal{H}_{L}^{1, \alpha}}=\left\|L^{\alpha / 2} f\right\|_{H_{L}^{1}}+\|f\|_{H_{L}^{1}} .
$$

Theorem 4.7. Let $\alpha>0, k \in \mathbf{N} \backslash\{0\}$ and $\lambda>d$. The following assertions are equivalent:

(i) $f \in \mathcal{H}_{L}^{1, \alpha}\left(\mathbf{R}^{d}\right)$;

(ii) $f \in H_{L}^{1}\left(\mathbf{R}^{d}\right)$ and $g_{k, \alpha}(f) \in L^{1}\left(\mathbf{R}^{d}\right)$ for $k>\alpha$;

(iii) $f \in H_{L}^{1}\left(\mathbf{R}^{d}\right)$ and $G_{k, \alpha}(f) \in L^{1}\left(\mathbf{R}^{d}\right)$ for $\alpha<k-(d+1) / 2$;

(iv) $f \in H_{L}^{1}\left(\mathbf{R}^{d}\right)$ and $g_{k, \alpha ; \lambda}^{*}(f) \in L^{1}\left(\mathbf{R}^{d}\right)$ for $\alpha<k-(d+1) / 2$.

Moreover, for every $f \in \mathcal{H}_{L}^{1, \alpha}\left(\mathbf{R}^{d}\right)$,

$$
\|f\|_{\mathcal{H}_{L}^{1, \alpha}} \sim\|f\|_{H_{L}^{1}}+\left\|g_{k, \alpha}(f)\right\|_{L^{1}} \sim\|f\|_{H_{L}^{1}}+\left\|G_{k, \alpha}(f)\right\|_{L^{1}} \sim\|f\|_{H_{L}^{1}}+\left\|g_{k, \alpha ; \lambda}^{*}(f)\right\|_{L^{1}}
$$

Since the above proof is similar to that of Theorem 1.7, we omit the details. Theorems 1.7 and 4.7 imply that the Hardy-Sobolev spaces $H_{L}^{1, \alpha}\left(\mathbf{R}^{d}\right)$ and $\mathcal{H}_{L}^{1, \alpha}\left(\mathbf{R}^{d}\right)$ are equivalent.

Acknowledgements. J.Z. Huang was supported by the Fundamental Research Funds for the Central Universities (No. 500419772). P. T. Li was supported by the National Natural Science Foundation of China under grants (No. 11871293, No. 11571217) and Shandong Natural Science Foundation of China (No. ZR2017JL008). Y. Liu was supported by the National Natural Science Foundation of China 
(No. 11671031), the Fundamental Research Funds for the Central Universities (No. FRF-BR-17-004B) and Beijing Municipal Science and Technology Project (No. Z17111000220000). J. Xin was supported by the National Natural Science Foundation of China (No. 11371183) and the Natural Science Foundation of Shandong Province (No. ZR2013AM004).

\section{References}

[1] Auscher, P., E. Russ, and P. Tchamitchian: Hardy Sobolev spaces on strongly Lipschitz domains of $\mathbf{R}^{n}$. - J. Funct. Anal. 218, 2005, 54-109.

[2] Auscher, P., and P. TChamitchian: Calcul fonctionnel précisé pour des opérateurs elliptiques complexes en dimension un (et applications à certaines équations elliptiques complexes en dimension deux). - Ann. Inst. Fourier (Grenoble) 45, 1995, 721-778.

[3] Badr, N., and F. Bernicot: Abstract Hardy-Sobolev spaces and interpolation. - J. Funct. Anal. 259, 2010, 1169-1208.

[4] BAdr, N., and G. DAfNi: Maximal characterization of Hardy-Sobolev spaces on manifolds. Contemp. Math. 545, 2011, 13-21.

[5] Betancor, J., J. Fariña, L. Rodríguez-Mesa, R. Testoni, and J. Torrea: Fractional square functions and potential spaces, II. - Acta Math. Sin. (English series) 33, 2015, 1759-1774.

[6] CAO, J., and D. YAnG: Hardy spaces $H_{L}^{p}\left(\mathbf{R}^{n}\right)$ associated with operators satisfying $k$-DaviesGaffney estimates. - Sci. China Math. 55, 2012, 1403-1440.

[7] CaO, J., D.-C. Chang, D. Yang, and S. Yang: Boundedness of second order Riesz transforms associated to Schrödinger operators on Musielak-Orlicz-Hardy spaces. - Commun. Pure Appl. Anal. 13, 2014, 1435-1463.

[8] Chang, D. C., G. Dafni, and E. M. Stein: Hardy spaces, BMO, and boundary value problems for the Laplacian on a smooth domain in $\mathbf{R}^{n}$. - Trans. Amer. Math. Soc. 351, 1999, 1605-1661.

[9] Cho, Y., and J. KIm: Atomic decomposition on Hardy-Sobolev spaces. - Studia Math. 177, 2006, 25-42.

[10] Coifman, R., P.-L. Lions, Y. Meyer, and S. Semmes: Compensated compactness and Hardy space. - J. Math. Pures Appl. 9, 1993, 247-286.

[11] Coifman, R., Y. Meyer, and E. M. Stein: Some new function spaces and their applications to harmonic analysis. - J. Funct. Anal. 62, 1985, 304-335.

[12] DeVore, R., and R. Sharpley: Maximal functions measuring smoothness. - Mem. Amer. Math. Soc. 47:293, 1984.

[13] DuOng, X., and L. YAn: Duality of Hardy and BMO spaces associated with operators with heat kernel bounds. - J. Amer. Math. Soc. 18, 2005, 943-973.

[14] Dziubański, J.: Spectral multiplier theorem for $H^{1}$ spaces associated with some Schrödinger operators. - Proc. Amer. Math. Soc. 12, 1999, 3605-3613.

[15] Dziubański, J., G. Garrigós, T. Martínez, J. Torrea, and J. Zienkiewicz: BMO spaces related to Schrödinger operator with potential satisfying reverse Hölder inequality. Math. Z. 249, 2005, 329-356.

[16] Dziubański, J., and J. Zienkiewicz: Hardy space $H^{1}$ associated to Schrödinger operator with potential satisfying reverse Hölder inequality. - Rev. Mat. Iberoam. 15, 1999, 279-296.

[17] Dziubański, J., and J. Zienkiewicz: $H^{p}$ spaces for Schrödinger operators. - In: Fourier Analysis and Ralated Topics, Vol. 56, Banach Center Publications, 2002, 45-53.

[18] Fefferman, C., and E. M. Stein: $H^{p}$ spaces of several variables. - Acta Math. 129, 1972, $137-193$.

[19] Fefferman, C., and E. M. Stein: Hardy spaces on homogeneous groups. - Princeton University Press, 1982. 
[20] Gatto, A., C. Segovia, and J. Jimnez: On the solution of the equation $\triangle^{m} F=f$ for $f \in H^{p}$. - In: Conference on harmonic analysis in honor of Antoni Zygmund, Vol. I, II (Chicago, Ill., 1981), 1983, 394-415.

[21] Hofmann, S., G. Lu, D. Mitrea, M. Mitrea, and L. Yan: Hardy spaces associated to non-negative self-adjoint operators satisfying Davies-Gaffney estimates. - Mem. Amer. Math. Soc. 214:1007, 2011.

[22] HuAng, J.: Hardy-Sobolev spaces associated with Hermite expansions and interpolation. Nonlinear Anal. 157, 2017, 104-122.

[23] Huang, J., W. Li, and Y. WAng: Hardy-Sobolev spaces associated with twisted convolution. - J. Funct. Spaces, Art. ID 5692746, 2017, 1-7.

[24] Janson, S.: On functions with derivatives in $H^{1}$. - In: Harmonic analysis and partial differential equations (El Escorial, 1987), Springer Lecture Notes in Math. 1384, 1989, 193-201.

[25] Jiang, R., and D. Yang: Orlicz-Hardy spaces associated with operators. - Sci. China Ser. A 52, 2009, 1042-1080.

[26] JiAng, R., and D. YANG: Orlicz-Hardy spaces associated with operators satisfying DaviesGaffney estimates. - Commun. Contemp. Math. 13, 2011, 331-373.

[27] JiAng, R., and D. YAng: Predual spaces of Banach completions of Orlicz-Hardy spaces associated with operators. - J. Fourier Anal. Appl. 17, 2011, 1-35.

[28] Koskela, P., and E. Saksman: Pointwise characterizations of Hardy-Sobolev functions. Math. Res. Lett. 15, 2008, 727-744.

[29] Lin, C., H. LiU, and Y. LiU: Hardy spaces associated with Schrödinger operators on the Heisenberg group. - arXiv:1106.4960 [math.AP].

[30] Lou, Z., and S. YAng: An atomic decomposition for the Hardy Sobolev space. - Taiwan J. Math. 11, 2007, 1167-1176.

[31] Ma, T., P. Stinga, J. Torrea, and C. Zhang: Regularity properties of Schrödinger operaors. - J. Math. Anal. Appl. 388, 2012, 817-837.

[32] Miyachi, A.: Hardy-Sobolev spaces and maximal functions. - J. Math. Soc. Japan 42, 1990, 73-90.

[33] OrobitG, J.: Spectral synthesis in spaces of functions with derivatives in $H^{1}$. - In: Harmonic analysis and partial differential equations (El Escorial, 1987), Springer Lecture Notes in Math. 1384, 1989, 202-206.

[34] Segovia, C., and R. Wheeden: On certain fractional area integrals. - J. Math. Mech. 19, 1969/1970, 247-262.

[35] Strichartz, R.: $H^{p}$ Sobolev spaces. - Colloq. Anal. 60-61:1, 1990, 129-139.

[36] Torchinsky, A.: Restrictions and extensions of potentials of $H^{p}$ distributions. - J. Funct. Anal. 31, 1979, 24-41.

[37] Torrea, J., and C. Zhang: Fractional vector-valued Littlewood-Paley-Stein theory for semigroups. - Proc. Roy. Soc. Edinburgh 144A, 2014, 637-667.

[38] YANG, D., and Y. Zhou: Localized Hardy spaces $H^{1}$ related to admissible functions on RD-spaces and applications to Schrödinger operators. - Trans. Amer. Math. Soc. 363, 2011, 1197-1239.

[39] YANG, D., and J. ZHANG: Variable Hardy spaces associated with operators satisfying DaviesGaffney estimates on metric measure spaces of homogeneous type. - Ann. Acad. Sci. Fenn. Math. 43, 2018, 47-87.

[40] YAnG, D., and S. YANG: Musielak-Orlicz-Hardy spaces associated with operators and their applications. - J. Geom. Anal. 24, 2014, 495-570.

[41] Zhang, J., D.-C. Chang, and D. YAng: Characterizations of Sobolev spaces associated to operators satisfying off-diagonal estimates on balls. - Math. Methods Appl. Sci. 40, 2017, $2907-2929$. 